\title{
Bogoyavlenskij symmetries of ideal MHD equilibria as Lie point transformations
}

\author{
Alexei F. Cheviakov \\ Department of Mathematics and Statistics, Queen's University, Kingston, K'7L 3N6 Canada \\ E-mail: alexch@mast.queensu.ca \\ Phone: $(+1)(613)$ 533-6000 x74464 \\ Fax: $(+1)(613)$ 533-2964
}

\begin{abstract}
In this paper we establish the correspondence between Bogoyavlenskij symmetries $[1,2]$ of the MHD equilibrium equations and Lie point transformations of these equations. We show that certain non-trivial Lie point transformations (that are obtained by direct application of Lie method) are equivalent to Bogoyavlenskij symmetries.
\end{abstract}

PACS Codes: 05.45.-a , 02.30.Jr, 02.90.+p, 52.30.Cv.

Keywords: MHD; plasma; PDE; Lie; symmetry.

\section{Introduction.}

Already for several decades the system of classical magnetohydrodynamics (MHD) equilibrium equations has been of great interest and importance for physicists working in different areas. Its direct applications include the problem of controlled thermonuclear fusion, astrophysical applications (star formation, solar activity) and terrestrial applications (laboratory and industrial plasmas, ball lightning models).

In the recent papers [1, 2] Bogoyavlenskij introduced new symmetry transforms of the ideal MHD equilibrium equations. In certain classes of plasma configurations, Bogoyavlenskij symmetries break geometrical symmetry, thus giving rise to important classes of non-symmetric MHD equilibrium solutions.

In this paper we study the possibility of finding complex intrinsic symmetries of systems of partial differential equations such as Bogoyavlenskij symmetries by applying a general method.

The goal of the paper is to prove that the Bogoyavlenskij symmetries are contained in particular Lie groups of point transformations, which are found independently using the classical Lie approach. 
The Bogoyavlenskij symmetries form an infinite-dimensional Abelian group of transformations with eight connected components in the case of incompressible plasmas, and four connected components in the case of compressible gas plasmas [2]. In Section 4 of the current paper, we prove that the system of MHD equilibrium equations in compressible and incompressible cases possesses specific infinite-dimensional Lie groups of point transformations, which are equivalent to Bogoyavlenskij symmetries.

The Lie symmetry method [3] used in this work is generally capable of detecting both simple geometric symmetries of systems of PDEs (e.g., rotations, scaling transforms and translations), and more complicated ones. When the Lie transformations are found, they can be used to build particular solutions of the system under consideration, to reduce the order and to obtain invariants. Self-similar solutions constructed from Lie symmetries often have transparent physical meaning. Many appropriate examples can be found in [4].

We remark, however, that not all symmetries of a given system can be found by the Lie method, but only continuous symmetries that have one-parametric Lie group structure.

Continuous Lie symmetries can also be used to obtain discrete symmetries of differential equations. One of the simplest ways of finding discrete symmetries is the complexification of the parameter (an example is Lemma 1 in Appendix C). A recently developed more powerful algorithm [5]-[7] enables the user to obtain all discrete point symmetries of systems of ordinary and partial differential equations. The algorithm proceeds by classifying the adjoint actions of discrete point symmetries on the Lie algebra of Lie point symmetry generators. This method is easy to apply and for simple systems does not require any computer algebra.

The advantage of the Lie group analysis procedure is that it can be applied directly to any system of equations (provided that all involved functions are sufficiently smooth).

On the other hand, the application of the Lie symmetry method is almost always extremely resource-demanding - it requires a lot of algebraic manipulation and the solution of large systems of dependent linear partial differential equations. This makes the analysis of systems of several PDEs in several variables "by hand" practically impossible. Due to this difficulty many important results obtained by the Lie method were discovered earlier using much less general techniques.

However, the use of modern analytical computation software often significantly facilitates the computations. Recently developed methods using Gröbner bases [8]-[11] and characteristic sets [12]-[13] to handle large overdetermined systems of partial differential equations, such as those arising from the Lie group analysis procedure, make it possible to perform complete or partial group analysis of many complicated systems.

A review of analytical computation software employing these ideas is given in [14].

In this work, the most involved algebraic manipulations were done on Waterloo Maple using Rif package for PDE systems reduction. This package is an extended version of well-known Standard Form package developed by Reid and Wittkopf [15].

Another widely used software package for Maple is diffgrob2 developed by E.L.Mansfield [16]. 


\section{MHD equilibrium equations and their symmetries.}

The classical description of equilibrium states of moving plasmas is given by the system of MHD equilibrium equations, which under the assumptions of infinite conductivity and negligible viscosity has the form [17]

$$
\begin{aligned}
& \rho \mathbf{V} \times \operatorname{curl} \mathbf{V}-\frac{1}{\mu} \mathbf{B} \times \operatorname{curl} \mathbf{B}-\operatorname{grad} P-\rho \operatorname{grad} \frac{\mathbf{V}^{2}}{2}=0, \\
& \operatorname{div} \rho \mathbf{V}=0, \quad \operatorname{curl}(\mathbf{V} \times \mathbf{B})=0, \quad \operatorname{div} \mathbf{B}=0 .
\end{aligned}
$$

Here $\mathbf{V}$ is plasma velocity; $\mathbf{B}$ is the vector of the magnetic field induction; $\rho$, plasma density; $P$, plasma pressure; and $\mu$, magnetic permeability coefficient.

We adopt the notation

$$
\mathbf{B}=\left(B_{1}, B_{2}, B_{3}\right), \mathbf{V}=\left(V_{1}, V_{2}, V_{3}\right)
$$

In the case of incompressible plasma, the equation

$$
\operatorname{div} \mathbf{V}=0
$$

is added to the above system; for a compressible case an appropriate equation of state must be chosen. For example, it can be the adiabatic ideal gas equation of state:

$$
P=\rho^{\gamma} \exp \left(S / c_{v}\right), \mathbf{V} \cdot \operatorname{grad} S=0
$$

Here $c_{v}$ is the heat capacity at constant volume; $\gamma$, the adiabatic exponent; and $S$, entropy.

Recently Bogoyavlenskij [1, 2] found that the ideal MHD equilibrium equations (1)-(3) possess the following symmetries.

Let $\{\mathbf{V}(\mathbf{r}), \mathbf{B}(\mathbf{r}), P(\mathbf{r}), \rho(\mathbf{r})\}$ be a solution of (1)-(3), where the density $\rho(\mathbf{r})$ is constant on both magnetic field lines and streamlines. Then $\left\{\mathbf{V}_{1}(\mathbf{r}), \mathbf{B}_{1}(\mathbf{r}), P_{1}(\mathbf{r}), \rho_{1}(\mathbf{r})\right\}$ is also a solution, where

$$
\begin{aligned}
& \mathbf{B}_{1}=b(\mathbf{r}) \mathbf{B}+c(\mathbf{r}) \sqrt{\mu \rho} \mathbf{V}, \\
& \mathbf{V}_{1}=\frac{c(\mathbf{r})}{a(\mathbf{r}) \sqrt{\mu \rho}} \mathbf{B}+\frac{b(\mathbf{r})}{a(\mathbf{r})} \mathbf{V}, \\
& \rho_{1}=a^{2}(\mathbf{r}) \rho, \quad P_{1}=C P+\left(C \mathbf{B}^{2}-\mathbf{B}_{1}^{2}\right) /(2 \mu) .
\end{aligned}
$$

Here

$$
b^{2}(\mathbf{r})-c^{2}(\mathbf{r})=C=\text { const },
$$

and $a(\mathbf{r}), b(\mathbf{r}), c(\mathbf{r})$ are functions constant on both magnetic field lines and streamlines (i.e. on magnetic surfaces $\Psi=$ const, when they exist).

These transformations form an infinite-dimensional Abelian group [2]

$$
G_{m}=A_{m} \oplus A_{m} \oplus R^{+} \oplus Z_{2} \oplus Z_{2} \oplus Z_{2},
$$


where $R^{+}$is a multiplicative group of positive numbers, and $A_{m}$ is an additive Abelian group of smooth functions in $\mathbb{R}^{3}$ that are constant on magnetic surfaces. The group $G_{m}$ has eight connected components.

Another transformation, which is applicable to compressible MHD equilibria (1)-(2), is given by the following formulas $[1,2]$ :

$$
\rho_{1}=a^{2}(\mathbf{r}) \rho, \quad \mathbf{B}_{1}=b \mathbf{B}, \quad \mathbf{V}_{1}=\frac{b}{a(\mathbf{r})} \mathbf{V}, \quad P_{1}=b^{2} P
$$

where $a(\mathbf{r})$ is an arbitrary smooth function that is constant on both magnetic field lines and streamlines, and $b \neq 0$ is a constant.

For the case of ideal gas with the equation of state (4), this transformation changes the entropy as follows:

$$
S_{1}=S+2 c_{v}(\ln |b|-\gamma \ln |a(\mathbf{r})|) .
$$

The symmetries (7)-(8) form the subgroup

$$
G_{0 m}=A_{m} \oplus R^{+} \oplus Z_{2} \oplus Z_{2}
$$

which has four connected components.

\section{Lie group formalism for the MHD equilibrium equa- tions.}

A solution to a system of $l$ first-order partial differential equations

$$
\begin{aligned}
& \mathbf{E}\left(\mathbf{x}, \mathbf{u}, \mathbf{u}_{1}\right)=0, \\
& \mathbf{E}=\left(E^{1}, \ldots, E^{l}\right), \mathbf{x}=\left(x^{1}, \ldots, x^{n}\right) \in X, \mathbf{u}=\left(u^{1}, \ldots, u^{m}\right) \in U, \\
& \underset{1}{\mathbf{u}}=\left(\frac{\partial u^{j}}{\partial x^{i}} \mid i=1, \ldots, n ; j=1, \ldots, m\right) \in U_{1}
\end{aligned}
$$

represents a manifold $\Omega$ in $(m+n)$ - dimensional space $X \times U$, which corresponds to a manifold $\Omega^{1}$ in $(m+n+m n)$ - dimensional prolonged (jet) space $X \times U \times U_{1}$ of dependent and independent variables together with partial derivatives [3].

Studying ideal MHD equilibria, one should take into account the generally the plasma domain is spanned by nested 2-dimensional magnetic surfaces - surfaces on which magnetic field lines and plasma streamlines lie [18].

In the case of adiabatic compressible MHD equilibrium equations, one has $n=3$ independent and $m=10$ dependent variables:

$$
\mathbf{x}=(x, y, z), \quad \mathbf{u}=\left(V_{1}, V_{2}, V_{3}, B_{1}, B_{2}, B_{3}, \Psi, P, \rho, S\right) .
$$

Here $\Psi$ is a function constant on magnetic field lines and plasma streamlines, i.e. on magnetic surfaces, when they exist:

$$
\operatorname{grad}(\Psi(\mathbf{r})) \cdot \mathbf{B}=0, \operatorname{grad}(\Psi(\mathbf{r})) \cdot \mathbf{V}=0 .
$$


The Lie method of seeking one-parametric groups of transformations that map solutions of (10) into solutions consists in finding the Lie algebra of vector fields tangent to the solution manifold $\Omega^{1}$ in the jet space. These vector fields serve as infinitesimal generators for a Lie symmetry group with representation

$$
\begin{array}{ll}
\left(x^{\prime}\right)^{i}=f^{i}(\mathbf{x}, \mathbf{u}, a) & (i=1, \ldots, n), \\
\left(u^{\prime}\right)^{j}=g^{j}(\mathbf{x}, \mathbf{u}, a) & (j=1, \ldots, m),
\end{array}
$$

and have the form

$$
\mathbf{v}=\sum_{i} \xi^{i}(\mathbf{x}, \mathbf{u}) \frac{\partial}{\partial x^{i}}+\sum_{k} \eta^{k}(\mathbf{x}, \mathbf{u}) \frac{\partial}{\partial u^{k}}+\sum_{i, k} \xi_{i}^{k}(\mathbf{x}, \mathbf{u}, \underset{1}{\mathbf{u}}) \frac{\partial}{\partial u_{i}^{k}} .
$$

Components of these tangent vector fields are expressed through the group representation as follows:

$$
\begin{aligned}
& \xi^{i}(\mathbf{x}, \mathbf{u})=\left.\frac{\partial f^{i}(\mathbf{x}, \mathbf{u}, a)}{\partial a}\right|_{a=0}, \quad \eta^{j}(\mathbf{x}, \mathbf{u})=\left.\frac{\partial g^{j}(\mathbf{x}, \mathbf{u}, a)}{\partial a}\right|_{a=0}, \\
& i=1, \ldots, n, \quad j=1, \ldots, m .
\end{aligned}
$$

The variables $\xi_{i}^{k}$ in (14) are the coordinates of the prolonged tangent vector field corresponding to the derivatives $u_{i}^{k}$ :

$$
\xi_{i}^{j}(\mathbf{x}, \mathbf{u}, \underset{1}{\mathbf{u}})=D_{i} \eta^{j}-\sum_{k=1}^{n} u_{k}^{j} D_{i} \xi^{k}, \quad D_{i} \equiv \frac{\partial}{\partial x^{i}}+\sum_{j=1}^{m} u_{i}^{j} \frac{\partial}{\partial u^{j}} .
$$

We remark that relation (16) defines an isomorphism between tangent vector fields (14) and infinitesimal operators

$$
X=\sum_{i} \xi^{i}(\mathbf{x}, \mathbf{u}) \frac{\partial}{\partial x^{i}}+\sum_{k} \eta^{k}(\mathbf{x}, \mathbf{u}) \frac{\partial}{\partial u^{k}} .
$$

The explicit reconstruction of the transformations (13) from a generator (14) is done by solving the initial value problem

$$
\begin{aligned}
& \frac{\partial f^{i}(a)}{\partial a}=\xi^{i}(\mathbf{f}, \mathbf{g}), \quad \frac{\partial g^{k}(a)}{\partial a}=\eta^{k}(\mathbf{f}, \mathbf{g}), \\
& f^{i}(0)=x^{i}, \quad g^{k}(0)=u^{k} .
\end{aligned}
$$

To find all Lie group generators admissible by the original system (10), one needs to solve the determining equations

$$
\left.\mathbf{v E}(\mathbf{x}, \mathbf{u}, \underset{1}{\mathbf{u}})\right|_{\mathbf{E}(\mathbf{x}, \mathbf{u}, \mathbf{u})=0}=0 .
$$

All $l$ determining equations (19) are linear partial differential equations with respect to $m+n$ unknown functions (15) of $m+n$ variables (11). 
According to the formula (19), the determining equations are obtained as follows. First, one applies the operator $\mathbf{v}$ to the original equations (10). Second, using the original equations as true equalities, one eliminates from this intermediate result some terms (usually the highest order partial derivatives).

To solve the determining equations and obtain the tangent vector field coordinates (15), one should use the fact that the latter do not depend on derivatives $u_{i}^{k}$. Therefore in all $l$ determining equations coefficients at different derivatives must equal zero. Thus the system (16) splits into $N \leq l(m n+1)$ simpler linear partial differential equations. In the case of adiabatic compressible plasma equilibria, for example, this generally leads to a system of 188 linear PDEs on 13 unknown functions.

It is not realistic to solve such a system "by hand"; however, computer algebra algorithms mentioned above can sometimes be successfully applied to reduce the system of equations and to exclude dependence of tangent vector field coordinates $\xi^{i}, \eta^{k}$ on some variables. It is shown in the proof of Theorem 1 how such a simplification can significantly reduce the system of determining equations to the point when it can be processed manually.

\section{Correspondence between Bogoyavlenskij symmetries and Lie transformations of the MHD equilibrium equations.}

In this section we answer the question about the possibility of obtaining the Bogoyavlenskij symmetries (5) and (7)-(8) of the MHD equilibrium equations using the Lie group formalism. This question was raised soon after the discovery of the symmetries.

Theorem 1 shows that the application of the Lie group formalism to MHD equilibria (1)-(2) yields certain Lie point transformations, some of which are infinite-dimensional.

Theorem 2 proves that these Lie point transformations are equivalent to the groups $G_{m}$ and $G_{0 m}$ of Bogoyavlenskij symmetries (for incompressible and compressible plasmas respectively).

Theorem 1 (i) Consider the incompressible MHD equilibrium system of equations (1)-(3), where the density $\rho(\mathbf{r})$ is constant on both magnetic field lines and streamlines. This system admits the infinitesimal operators

$$
\begin{aligned}
& X^{(1)}=M(\mathbf{r})\left(\sum_{k=1}^{3} \frac{B_{k}}{\mu \rho} \frac{\partial}{\partial V_{k}}+\sum_{k=1}^{3} V_{k} \frac{\partial}{\partial B_{k}}-\frac{1}{\mu}(\mathbf{V} \cdot \mathbf{B}) \frac{\partial}{\partial P}\right), \\
& X^{(2)}=\sum_{k=1}^{3} V_{k} \frac{\partial}{\partial V_{k}}+\sum_{k=1}^{3} B_{k} \frac{\partial}{\partial B_{k}}+2 P \frac{\partial}{\partial P}, \\
& X^{(3)}=N(\mathbf{r})\left(2 \rho \frac{\partial}{\partial \rho}-\sum_{k=1}^{3} V_{k} \frac{\partial}{\partial V_{k}}\right), \\
& X^{(4)}=\frac{\partial}{\partial P} .
\end{aligned}
$$


These operators form a basis of the Lie algebra of infinitesimal operators in the class of Lie point transformations $\left\{\mathbf{x}^{\prime}=\mathbf{x}, \mathbf{u}^{\prime}=\mathbf{g}(\mathbf{u}, a)\right\}$. Here $M(\mathbf{r}), N(\mathbf{r})$ are arbitrary smooth functions constant on both magnetic field lines and streamlines.

(ii) Compressible ideal MHD equilibrium equations (1)-(2) with ideal gas state equation (4), for arbitrary density, admit the infinitesimal operators

$$
\begin{aligned}
& X^{(5)}=\sum_{k=1}^{3} V_{k} \frac{\partial}{\partial V_{k}}+\sum_{k=1}^{3} B_{k} \frac{\partial}{\partial B_{k}}+2 P \frac{\partial}{\partial P}+2 c_{v} \frac{\partial}{\partial S}, \\
& X^{(6)}=N(\mathbf{r})\left(2 \rho \frac{\partial}{\partial \rho}-\sum_{k=1}^{3} V_{k} \frac{\partial}{\partial V_{k}}-2 c_{v} \gamma \frac{\partial}{\partial S}\right),
\end{aligned}
$$

where $N(\mathbf{r})$ is an arbitrary smooth function constant on both magnetic field lines and streamlines.

The proof of Theorem 1 is given in the Appendix A. It directly follows the Lie group analysis procedure. In the alternative proof of the theorem, operators (20)-(22), (24)-(25) are obtained by direct differentiation of Bogoyavlenskij symmetries (5), (7) with respect to a properly chosen parameter, as shown in Appendix B. This alternative proof is simpler, but it is based on the knowledge of the precise form of Bogoyavlenskij symmetries, while the original proof does not require it.

\section{Remark.}

Let us explicitly write down the transformations contained in the infinitesimal operators (20)-(25).

According to the reconstruction procedure (18), for the operator (20), we have

$$
\rho_{1}=\rho, \quad \mathbf{x}_{1}=\mathbf{x},
$$

and need to solve the linear initial value problem

$$
\begin{aligned}
& \frac{\partial \mathbf{V}_{1}}{\partial \tau}=\mathbf{B}_{1} \frac{M(\mathbf{r})}{\mu \rho}, \quad \frac{\partial \mathbf{B}_{1}}{\partial \tau}=\mathbf{V}_{1} M(\mathbf{r}), \quad \frac{\partial P_{1}}{\partial \tau}=-\frac{M(\mathbf{r})}{\mu}\left(\mathbf{V}_{1} \cdot \mathbf{B}_{1}\right), \\
& \mathbf{V}_{1}(\tau=0)=\mathbf{V}, \quad \mathbf{B}_{1}(\tau=0)=\mathbf{B}, \quad P_{1}(\tau=0)=P .
\end{aligned}
$$

The solution is

$$
\begin{aligned}
& \mathbf{B}_{1}=\cosh \left(\frac{M(\mathbf{r}) \tau}{\sqrt{\mu \rho}}\right) \mathbf{B}+\sinh \left(\frac{M(\mathbf{r}) \tau}{\sqrt{\mu \rho}}\right) \sqrt{\mu \rho} \mathbf{V} \\
& \mathbf{V}_{1}=\sinh \left(\frac{M(\mathbf{r}) \tau}{\sqrt{\mu \rho}}\right) \frac{\mathbf{B}}{\sqrt{\mu \rho}}+\cosh \left(\frac{M(\mathbf{r}) \tau}{\sqrt{\mu \rho}}\right) \mathbf{V} \\
& P_{1}=P+\left(\mathbf{B}^{2}-\mathbf{B}_{1}^{2}\right) /(2 \mu), \quad \rho_{1}=\rho .
\end{aligned}
$$

The infinitesimal operator (20) thus contains the possibility of "mixing" the components of the vector fields $\mathbf{B}$ and $\mathbf{V}$ of the original solution into a new solution. 
The same way by solving a corresponding initial value problem (18) we find that transformations contained in the operator (21) are scalings

$$
\rho_{1}=\rho, \quad \mathbf{B}_{1}=\exp (\tau) \mathbf{B}, \quad \mathbf{V}_{1}=\exp (\tau) \mathbf{V}, \quad P_{1}=\exp (2 \tau) P
$$

the operator (22) corresponds to infinite-dimensional scalings

$$
\rho_{1}=\exp (2 N(\mathbf{r}) \tau) \rho, \quad \mathbf{B}_{1}=\mathbf{B}, \quad \mathbf{V}_{1}=\exp (-N(\mathbf{r}) \tau) \mathbf{V}, \quad P_{1}=P
$$

the operator $(23)$ - to translations

$$
\rho_{1}=\rho, \quad \mathbf{B}_{1}=\mathbf{B}, \quad \mathbf{V}_{1}=\mathbf{V}, \quad P_{1}=P+\tau .
$$

The transformations provided by the operators (24) and (25) are respectively

$$
\rho_{1}=\rho, \quad \mathbf{B}_{1}=\exp (\tau) \mathbf{B}, \quad \mathbf{V}_{1}=\exp (\tau) \mathbf{V}, \quad P_{1}=\exp (2 \tau) P, \quad S_{1}=S+2 c_{v} \tau
$$

and

$$
\rho_{1}=\exp (2 N(\mathbf{r}) \tau) \rho, \quad \mathbf{B}_{1}=\mathbf{B}, \quad \mathbf{V}_{1}=\exp (N(\mathbf{r}) \tau) \mathbf{V}, P_{1}=P, \quad S_{1}=S-2 c_{v} \gamma N(\mathbf{r}) \tau
$$

Theorem 2 (i) Lie point transformations (27)-(29) are equivalent to the group $G_{m}$ of Bogoyavlenskij transformations (5), (6).

(ii) Lie point transformations (31)- (32) are equivalent to the group $G_{0 m}$ of Bogoyavlenskij transformations (7)-(8), (9).

The proof of Theorem 2 is presented in the Appendix C.

\section{Summary}

It is remarkable that the infinite-dimensional groups of Bogoyavlenskij symmetries (5), (7)(8) of the MHD equilibrium equations (1)-(2), the richest known class of transformations for these equations, is implied by the Lie point transformations of these equations.

Bogoyavlenskij symmetries form infinite-dimensional Abelian groups: $G_{m}=A_{m} \oplus A_{m} \oplus$ $R^{+} \oplus Z_{2} \oplus Z_{2} \oplus Z_{2}$ in the incompressible case and $G_{0 m}=A_{m} \oplus R^{+} \oplus Z_{2} \oplus Z_{2}$ in the compressible case. $G_{m}$ has eight connected components, and $G_{0 m}$ has four. In this paper we have shown that the groups $G_{m}$ and $G_{0 m}$ are equivalent to Lie point transformations generated by infinitesimal operators (20)-(22) and (24)-(25) respectively.

Thus Bogoyavlenskij symmetries are obtained from the standard procedure of Lie group analysis that is applicable to any system of PDEs with sufficiently smooth coefficients.

The Lie point transformations that correspond to Bogoyavlenskij symmetries were found by direct application of the Lie procedure to the MHD equilibrium equations (1)-(2) in incompressible (3) and compressible (4) cases. 
The Lie procedure in application to MHD equilibria is described in Section 3. Every solution to a system of PDEs with $n$ variables and $m$ unknown functions represents a manifold $\Omega^{1}$ in $(m+n+m n)$ - dimensional jet space $X \times U \times U_{1}$ of independent and dependent variables $\mathbf{x}, \mathbf{u}$ (11) and partial derivatives $u_{i}^{k}(10)$. The Lie procedure consists in finding vector fields $\mathbf{v}(14)$ tangent to $\Omega^{1}$. These vector fields serve as infinitesimal transformation group generators. Their components $\xi^{i}, \eta^{j}(15)$ are functions of all independent and dependent variables. The equations (19) for determining the tangent vector field components are the conditions of invariance of the solution manifold $\Omega^{1}$ under the action of $\mathbf{v}$.

It is known that generally the ideal plasma domain is spanned by nested 2-dimensional magnetic surfaces - surfaces tangent to plasma velocity and magnetic field [18]. In the group analysis procedure, this fact was taken into account by explicitly introducing a function $\Psi(\mathbf{r})$ (12) constant on every magnetic surface (or on magnetic field lines and plasma streamlines, if the surfaces seize to exist.) Introducing this function enables one to find Lie symmetries depending on functions constant magnetic surfaces.

The determining equations (19) are linear first-order partial differential equations. They are solved by employing the fact that the tangent vector field components do not depend on partial derivatives. Thus for the case of incompressible MHD equilibrium the determining system splits into 150 equations on 11 unknown functions, in the compressible case - into 188 equations on 13 unknown functions. Handling these systems, even with the help of computer symbolic manipulation software described in the introduction, puts extremely high demands on computer resources. Therefore we restricted our study to a subgroup of Lie point transformations of the type $\left\{\mathbf{x}^{\prime}=\mathbf{x} ; \quad \mathbf{u}^{\prime}=\mathbf{g}(\mathbf{u}, a)\right\}$. These transformations preserve spatial variables and do not depend on them. In this case we got 141 determining equations for the incompressible case, and 187 - for the compressible case. These systems are substantially simpler than those arising from the general Lie procedure. Using Maple with Rif package, the systems were reduced respectively to 21 and 10 equations (in the compressible case, additional simplifying assumptions had to be used). Solving them, we obtained the transformation generators (20)-(23) for incompressible MHD equilibria, and (24)-(25) for compressible MHD equilibria.

The operators (20)-(23) admissible by incompressible MHD equilibria form a basis of the Lie algebra of infinitesimal operators corresponding to the subgroup $\left\{\mathbf{x}^{\prime}=\mathbf{x}, \mathbf{u}^{\prime}=\mathbf{g}(\mathbf{u}, a)\right\}$ of the group (13) of all Lie point transformations.

Theorem 2 stated above shows that the transformations generated by operators (20)-(22), (24)-(25) are equivalent to Bogoyavlenskij symmetries $G_{m}, G_{0 m}$.

This result illustrates that the general Lie approach of analyzing systems of partial differential equations is capable of revealing highly non-trivial intrinsic transformations, that may have great importance in applications, as is the case for Bogoyavlenskij symmetries.

The complete Lie group analysis of compressible and incompressible MHD equilibrium equations, taking into account the specific topology (general existence of magnetic surfaces), together with determination of discrete point symmetries using their adjoint action on the Lie algebra of symmetry generators (see the introduction), will be addressed in a subsequent paper.

The author thanks Leo B. Jonker for useful discussion. 


\section{A Proof of Theorem 1}

\section{Proof.}

(i) First, we prove that the operators $X^{(1)}-X^{(4)}$ are admissible for incompressible MHD equilibria with density $\rho(\mathbf{r})$ constant on magnetic field lines and streamlines. The complete system of equations under consideration can be written as follows:

$$
\begin{aligned}
& \rho \mathbf{V} \times(\operatorname{curl} \mathbf{V})-\frac{1}{\mu} \mathbf{B} \times(\operatorname{curl} \mathbf{B})-\operatorname{grad} P-\rho \operatorname{grad} \frac{\mathbf{V}^{2}}{2}=0, \\
& \operatorname{div} \mathbf{B}=0, \operatorname{div} \mathbf{V}=0, \operatorname{curl}(\mathbf{V} \times \mathbf{B})=0, \\
& \rho(\mathbf{r})=\rho(\Psi(\mathbf{r})), \quad \operatorname{grad}(\Psi(\mathbf{r})) \cdot \mathbf{B}=0, \operatorname{grad}(\Psi(\mathbf{r})) \cdot \mathbf{V}=0 .
\end{aligned}
$$

Here $\Psi(\mathbf{r})$ is an arbitrary function constant on magnetic field lines and streamlines (hence on magnetic surfaces, when they exist).

The system (33)-(35) consists of $l=10$ equations. It has $n=3$ independent and $m=8$ dependent variables:

$$
\mathbf{x}=(x, y, z), \quad \mathbf{u}=\left(V_{1}, V_{2}, V_{3}, B_{1}, B_{2}, B_{3}, \Psi, P\right) .
$$

Let us apply the Lie procedure described in Section 3, assuming that the transformations do not depend on spatial variables, and that the spatial variables themselves are not transformed. Thus we are looking for the transformations of the type

$$
\begin{aligned}
& \left(x^{\prime}\right)^{i}=f^{i}(\mathbf{x}, \mathbf{u}, a) \equiv x^{i} ; \quad\left(u^{\prime}\right)^{k}=g^{k}(\mathbf{x}, \mathbf{u}, a) \equiv g^{k}(\mathbf{u}, a) ; \\
& i=1, \ldots, 3, \quad k=1, \ldots, 8 .
\end{aligned}
$$

They form a subgroup of the general Lie group of transformations (13).

Remark. Without the assumptions (37), handling the computations takes significantly longer time and puts much higher demands on computer resources. The problem of performing the complete group analysis of the MHD equilibrium system with density constant on magnetic filed field lines and streamlines (33)-(35) is therefore out of the scope of this work and will be addressed in a subsequent paper.

The unknown quantities to be found are the tangent vector field coordinates

$$
\eta^{k}(\mathbf{u})=\left.\frac{\partial g^{k}(\mathbf{u}, a)}{\partial a}\right|_{a=0}, \quad k=1, \ldots, 8
$$

(We have $\xi^{i}(\mathbf{x}, \mathbf{u})=\left.\frac{\partial f^{i}(\mathbf{x}, \mathbf{u}, a)}{\partial a}\right|_{a=0}=0, i=1, \ldots, 3$.)

Applying the corresponding prolonged $\mathbf{v}$ (14) to every equation of the system (33)-(35), we get the following system of equations:

$$
\begin{aligned}
& \xi_{2}^{4} B_{2}+\xi_{3}^{4} B_{3}-\xi_{1}^{8}+\eta^{5}\left(-\partial B_{2} / \partial x+\partial B_{1} / \partial y\right)+\eta^{6}\left(\partial B_{1} / \partial z-\partial B_{3} / \partial x\right)- \\
& \xi_{1}^{5} B_{2}-\xi_{1}^{6} B_{3}-\eta^{2} \rho(\Psi) \partial V_{1} / \partial y-\eta^{3} \rho(\Psi) \partial V_{1} / \partial z-\xi_{1}^{1} \rho(\Psi) V_{1}-\xi_{2}^{1} \rho(\Psi) V_{2}-\xi_{3}^{1} \rho(\Psi) V_{3} \\
& -\eta^{7}\left(V_{1} \partial V_{1} / \partial x+V_{2} \partial V_{1} / \partial y+V_{3} \partial V_{1} / \partial z\right) d \rho(\Psi) / d \Psi-\eta^{1} \rho(\Psi) \partial V_{1} / \partial x=0,
\end{aligned}
$$




$$
\begin{aligned}
& \xi_{1}^{5} B_{1}+\xi_{3}^{5} B_{3}-\xi_{2}^{8}+\eta^{4}\left(\partial B_{2} / \partial x-\partial B_{1} / \partial y\right)+\eta^{6}\left(-\partial B_{3} / \partial y+\partial B_{2} / \partial z\right)- \\
& \eta^{1} \rho(\Psi) \partial V_{2} / \partial x-\eta^{3} \rho(\Psi) \partial V_{2} / \partial z-\eta^{7}\left(V_{1} \partial V_{2} / \partial x+V_{2} \partial V_{2} / \partial y+V_{3} \partial V_{2} / \partial z\right) d \rho(\Psi) / d \Psi \\
& -\xi_{1}^{2} \rho(\Psi) V_{1}-\xi_{2}^{2} \rho(\Psi) V_{2}-\xi_{3}^{2} \rho(\Psi) V_{3}-\xi_{2}^{4} B_{1}-\xi_{2}^{6} B_{3}-\eta^{2} \rho(\Psi) \partial V_{2} / \partial y=0 \\
& \quad \xi_{1}^{6} B_{1}+\xi_{2}^{6} B_{2}-\xi_{3}^{8}-\eta^{1} \rho(\Psi) \partial V_{3} / \partial x-\eta^{2} \rho(\Psi) \partial V_{3} / \partial y-\eta^{3} \rho(\Psi) \partial V_{3} / \partial z-\xi_{1}^{3} \rho(\Psi) V_{1} \\
& \quad-\eta^{7}\left(V_{1} \partial V_{3} / \partial x+V_{2} \partial V_{3} / \partial y+V_{3} \partial V_{3} / \partial z\right) d \rho(\Psi) / d \Psi-\xi_{2}^{3} \rho(\Psi) V_{2}-\xi_{3}^{3} \rho(\Psi) V_{3} \\
& \quad+\eta^{4}\left(-\partial B_{1} / \partial z+\partial B_{3} / \partial x\right)+\eta^{5}\left(\partial B_{3} / \partial y-\partial B_{2} / \partial z\right)-\xi_{3}^{4} B_{1}-\xi_{3}^{5} B_{2}=0, \\
& \quad \xi_{1}^{4}+\xi_{2}^{5}+\xi_{3}^{6}=0 \\
& \quad \xi_{1}^{1}+\xi_{2}^{2}+\xi_{3}^{3}=0, \\
& \quad \eta^{4}\left(-\partial V_{2} / \partial y-\partial V_{3} / \partial z\right)+\eta^{5} \partial V_{1} / \partial y+\eta^{6} \partial V_{1} / \partial z \\
& \quad+\eta^{1}\left(\partial B_{2} / \partial y+\partial B_{3} / \partial z\right)-\eta^{2} \partial B_{1} / \partial y-\eta^{3} \partial B_{1} / \partial z \\
& \quad+\xi_{2}^{5} V_{1}+\xi_{3}^{6} V_{1}+\xi_{2}^{1} B_{2}+\xi_{3}^{1} B_{3}-\xi_{2}^{4} V_{2}-\xi_{2}^{2} B_{1}-\xi_{3}^{3} B_{1}-\xi_{3}^{4} V_{3}=0 \\
& \quad \eta^{5}\left(-\partial V_{3} / \partial z-\partial V_{1} / \partial x\right)+\eta^{6} \partial V_{2} / \partial z+\eta^{4} \partial V_{2} / \partial x \\
& \quad+\eta^{2}\left(\partial B_{3} / \partial z+\partial B_{1} / \partial x\right)-\eta^{1} \partial B_{2} / \partial x-\eta^{3} \partial B_{2} / \partial z \\
& \quad+\xi_{1}^{4} V_{2}+\xi_{3}^{6} V_{2}+\xi_{1}^{2} B_{1}+\xi_{3}^{2} B_{3}-\xi_{3}^{5} V_{3}-\xi_{1}^{1} B_{2}-\xi_{3}^{3} B_{2}-\xi_{1}^{5} V_{1}=0 \\
& \quad \eta^{6}\left(-\partial V_{2} / \partial y-\partial V_{1} / \partial x\right)+\eta^{4} \partial V_{3} / \partial x+\eta^{5} \partial V_{3} / \partial y \\
& \quad+\eta^{3}\left(\partial B_{1} / \partial x+\partial B_{2} / \partial y\right)-\eta^{1} \partial B_{3} / \partial x-\eta^{2} \partial B_{3} / \partial y \\
& \quad+\xi_{1}^{4} V_{3}+\xi_{2}^{5} V_{3}+\xi_{1}^{3} B_{1}+\xi_{2}^{3} B_{2}-\xi_{1}^{6} V_{1}-\xi_{2}^{6} V_{2}-\xi_{1}^{1} B_{3}-\xi_{2}^{2} B_{3}=0 \\
& \quad \eta^{4} \partial \Psi / \partial x+\eta^{5} \partial \Psi / \partial y+\eta^{6} \partial \Psi / \partial z+\xi_{1}^{7} B_{1}+\xi_{2}^{7} B_{2}+\xi_{3}^{7} B_{3}=0 \\
& \eta^{1} \partial \Psi / \partial x+\eta^{2} \partial \Psi / \partial y+\eta^{3} \partial \Psi / \partial z+\xi_{1}^{7} V_{1}+\xi_{2}^{7} V_{2}+\xi_{3}^{7} V_{3}=0 . \\
&
\end{aligned}
$$

According to (19), we need to solve the above ten determining equations under the condition that the original equations are also satisfied. For this purpose, we express ten derivatives

$$
\frac{\partial \Psi}{\partial x}, \frac{\partial \Psi}{\partial y}, \frac{\partial B_{3}}{\partial z}, \frac{\partial V_{1}}{\partial x}, \frac{\partial V_{2}}{\partial x}, \frac{\partial V_{3}}{\partial x}, \frac{\partial V_{3}}{\partial z}, \frac{\partial P}{\partial x}, \frac{\partial P}{\partial y}, \frac{\partial P}{\partial z}
$$

from the system (33)-(35) and substitute them, together with explicitly written prolonged vector field coordinates (16), into (39)-(48).

To solve the resulting system, and obtain the tangent vector field coordinates (38), one should use the fact that the latter do not depend on derivatives $u_{i}^{k}$. Setting in all ten determining equations the coefficients at different derivatives to zero, we get 141 dependent partial differential equations on 8 unknown functions $\eta^{1}, . ., \eta^{8}$.

Using the Rif package in Waterloo Maple software to reduce this system, we obtain the following equations:

$$
\begin{aligned}
& \eta^{1}=\eta^{1}\left(V_{1}, B_{1}, \Psi\right), \eta^{2}=\eta^{2}\left(V_{2}, B_{2}, \Psi\right), \eta^{3}=\eta^{3}\left(V_{3}, B_{3}, \Psi\right) \\
& \eta^{4}=\eta^{4}\left(V_{1}, B_{1}, \Psi\right), \eta^{5}=\eta^{5}\left(V_{2}, B_{2}, \Psi\right), \eta^{6}=\eta^{6}\left(V_{3}, B_{3}, \Psi\right) \\
& \eta^{7}=\eta^{7}(\Psi)
\end{aligned}
$$


When these equations are substituted into the original system, it reduces to as few as 21 independent equations, which are integrated by hand to give the infinitesimal operator

$$
\begin{aligned}
& X=\sum_{k=1}^{3}\left(M(\Psi) \frac{B_{k}}{\mu \rho}+\left(C_{1}-N(\Psi)\right) V_{k}\right) \frac{\partial}{\partial V_{k}}+\sum_{k=1}^{3}\left(M(\Psi) V_{k}+C_{1} B_{k}\right) \frac{\partial}{\partial B_{k}} \\
& +\left(-\frac{1}{\mu}(\mathbf{V} \cdot \mathbf{B}) M(\Psi)+2 C_{1} P+C_{2}\right) \frac{\partial}{\partial P}+2 \rho N(\Psi) \frac{\partial}{\partial \rho} .
\end{aligned}
$$

Here $M(\Psi)=M(\Psi(\mathbf{r})), N(\Psi)=N(\Psi(\mathbf{r}))$ are arbitrary functions constant on magnetic field lines and streamlines, and $C_{1}, C_{2}$ are free constants.

The operator (50) is evidently a general linear combination of infinitesimal operators (20)(23).

We now verify that they form a Lie algebra basis. Indeed, their commutators are

$$
\begin{aligned}
& {\left[X^{(1)}, X^{(2)}\right]=\left[X^{(1)}, X^{(4)}\right]=\left[X^{(2)}, X^{(3)}\right]=\left[X^{(3)}, X^{(4)}\right]=0,} \\
& {\left[X^{(2)}, X^{(4)}\right]=-2 X^{(4)},} \\
& {\left[X^{(1)}, X^{(3)}\right]=Q(\Psi) X^{(1)},}
\end{aligned}
$$

where

$$
Q(\Psi)=N(r)-2 \rho(\Psi) \frac{\partial M(\Psi)}{\partial \Psi} / M(\Psi) \frac{\partial \rho(\Psi)}{\partial \Psi} .
$$

Thus the part (i) of the theorem is proven.

(ii) Now we show that the operators $X^{(5)}, X^{(6)}(24)$ - (25) are admissible for compressible MHD equilibria with the ideal gas equation of state and entropy constant along the streamlines. The complete system of equations under consideration in this case is

$$
\begin{aligned}
& \rho \mathbf{V} \times(\operatorname{curl} \mathbf{V})-\frac{1}{\mu} \mathbf{B} \times(\operatorname{curl} \mathbf{B})-\operatorname{grad} P-\rho \operatorname{grad} \frac{\mathbf{V}^{2}}{2}=0, \\
& \operatorname{div} \mathbf{B}=0, \operatorname{div} \rho \mathbf{V}=0, \operatorname{curl}(\mathbf{V} \times \mathbf{B})=0 \\
& \operatorname{grad} \Psi(\mathbf{r}) \cdot \mathbf{B}=0, \operatorname{grad} \Psi(\mathbf{r}) \cdot \mathbf{V}=0, \\
& P=\rho^{\gamma} \exp \left(S / c_{v}\right), \operatorname{grad} S \cdot \mathbf{V}=0 .
\end{aligned}
$$

Here $\Psi(\mathbf{r})$ is an arbitrary function constant on magnetic field lines and streamlines. It is needed because the function $N(\mathbf{r})$ with the same properties enters into $X^{(2)}$.

The system (51)-(54) consists of $l=12$ equations. It has $n=3$ independent and $m=10$ dependent variables:

$$
\mathbf{x}=(x, y, z), \quad \mathbf{u}=\left(V_{1}, V_{2}, V_{3}, B_{1}, B_{2}, B_{3}, \Psi, P, \rho, S\right) .
$$

In a manner parallel to that in (i), we look for a subgroup of Lie point transformations of the type (37). Applying the operator $\mathbf{v}$ (14) to every equation of the system under consideration, we get the following system of equations: 


$$
\begin{aligned}
& \xi_{2}^{4} B_{2}+\xi_{3}^{4} B_{3}-\xi_{1}^{8}+\eta^{5}\left(-\partial B_{2} / \partial x+\partial B_{1} / \partial y\right)+\eta^{6}\left(\partial B_{1} / \partial z-\partial B_{3} / \partial x\right)- \\
& \xi_{1}^{5} B_{2}-\xi_{1}^{6} B_{3}-\eta^{2} \rho(\Psi) \partial V_{1} / \partial y-\eta^{3} \rho(\Psi) \partial V_{1} / \partial z-\xi_{1}^{1} \rho(\Psi) V_{1}-\xi_{2}^{1} \rho(\Psi) V_{2}-\xi_{3}^{1} \rho(\Psi) V_{3} \\
& -\eta^{7}\left(V_{1} \partial V_{1} / \partial x+V_{2} \partial V_{1} / \partial y+V_{3} \partial V_{1} / \partial z\right) d \rho(\Psi) / d \Psi-\eta^{1} \rho(\Psi) \partial V_{1} / \partial x=0, \\
& \xi_{1}^{5} B_{1}+\xi_{3}^{5} B_{3}-\xi_{2}^{8}+\eta^{4}\left(\partial B_{2} / \partial x-\partial B_{1} / \partial y\right)+\eta^{6}\left(-\partial B_{3} / \partial y+\partial B_{2} / \partial z\right)- \\
& \eta^{1} \rho(\Psi) \partial V_{2} / \partial x-\eta^{3} \rho(\Psi) \partial V_{2} / \partial z-\eta^{7}\left(V_{1} \partial V_{2} / \partial x+V_{2} \partial V_{2} / \partial y+V_{3} \partial V_{2} / \partial z\right) d \rho(\Psi) / d \Psi \\
& -\xi_{1}^{2} \rho(\Psi) V_{1}-\xi_{2}^{2} \rho(\Psi) V_{2}-\xi_{3}^{2} \rho(\Psi) V_{3}-\xi_{2}^{4} B_{1}-\xi_{2}^{6} B_{3}-\eta^{2} \rho(\Psi) \partial V_{2} / \partial y=0 \\
& \xi_{1}^{6} B_{1}+\xi_{2}^{6} B_{2}-\xi_{3}^{8}-\eta^{1} \rho(\Psi) \partial V_{3} / \partial x-\eta^{2} \rho(\Psi) \partial V_{3} / \partial y-\eta^{3} \rho(\Psi) \partial V_{3} / \partial z-\xi_{1}^{3} \rho(\Psi) V_{1} \\
& -\eta^{7}\left(V_{1} \partial V_{3} / \partial x+V_{2} \partial V_{3} / \partial y+V_{3} \partial V_{3} / \partial z\right) d \rho(\Psi) / d \Psi-\xi_{2}^{3} \rho(\Psi) V_{2}-\xi_{3}^{3} \rho(\Psi) V_{3} \\
& +\eta^{4}\left(-\partial B_{1} / \partial z+\partial B_{3} / \partial x\right)+\eta^{5}\left(\partial B_{3} / \partial y-\partial B_{2} / \partial z\right)-\xi_{3}^{4} B_{1}-\xi_{3}^{5} B_{2}=0 \\
& \xi_{1}^{4}+\xi_{2}^{5}+\xi_{3}^{6}=0 \\
& \rho\left(\xi_{1}^{1}+\xi_{2}^{2}+\xi_{3}^{3}\right)+V_{1} \xi_{1}^{9}+V_{2} \xi_{2}^{9}+V_{3} \xi_{3}^{9}=0, \\
& \eta^{4}\left(-\partial V_{2} / \partial y-\partial V_{3} / \partial z\right)+\eta^{5} \partial V_{1} / \partial y+\eta^{6} \partial V_{1} / \partial z \\
& +\eta^{1}\left(\partial B_{2} / \partial y+\partial B_{3} / \partial z\right)-\eta^{2} \partial B_{1} / \partial y-\eta^{3} \partial B_{1} / \partial z \\
& +\xi_{2}^{5} V_{1}+\xi_{3}^{6} V_{1}+\xi_{2}^{1} B_{2}+\xi_{3}^{1} B_{3}-\xi_{2}^{4} V_{2}-\xi_{2}^{2} B_{1}-\xi_{3}^{3} B_{1}-\xi_{3}^{4} V_{3}=0, \\
& \eta^{5}\left(-\partial V_{3} / \partial z-\partial V_{1} / \partial x\right)+\eta^{6} \partial V_{2} / \partial z+\eta^{4} \partial V_{2} / \partial x \\
& +\eta^{2}\left(\partial B_{3} / \partial z+\partial B_{1} / \partial x\right)-\eta^{1} \partial B_{2} / \partial x-\eta^{3} \partial B_{2} / \partial z \\
& +\xi_{1}^{4} V_{2}+\xi_{3}^{6} V_{2}+\xi_{1}^{2} B_{1}+\xi_{3}^{2} B_{3}-\xi_{3}^{5} V_{3}-\xi_{1}^{1} B_{2}-\xi_{3}^{3} B_{2}-\xi_{1}^{5} V_{1}=0, \\
& \eta^{6}\left(-\partial V_{2} / \partial y-\partial V_{1} / \partial x\right)+\eta^{4} \partial V_{3} / \partial x+\eta^{5} \partial V_{3} / \partial y \\
& +\eta^{3}\left(\partial B_{1} / \partial x+\partial B_{2} / \partial y\right)-\eta^{1} \partial B_{3} / \partial x-\eta^{2} \partial B_{3} / \partial y \\
& +\xi_{1}^{4} V_{3}+\xi_{2}^{5} V_{3}+\xi_{1}^{3} B_{1}+\xi_{2}^{3} B_{2}-\xi_{1}^{6} V_{1}-\xi_{2}^{6} V_{2}-\xi_{1}^{1} B_{3}-\xi_{2}^{2} B_{3}=0, \\
& \eta^{4} \partial \Psi / \partial x+\eta^{5} \partial \Psi / \partial y+\eta^{6} \partial \Psi / \partial z+\xi_{1}^{7} B_{1}+\xi_{2}^{7} B_{2}+\xi_{3}^{7} B_{3}=0, \\
& \eta^{1} \partial \Psi / \partial x+\eta^{2} \partial \Psi / \partial y+\eta^{3} \partial \Psi / \partial z+\xi_{1}^{7} V_{1}+\xi_{2}^{7} V_{2}+\xi_{3}^{7} V_{3}=0, \\
& \eta^{8}-\left(\gamma \rho^{\gamma-1} \eta^{9}+\rho^{\gamma} \eta^{10} / c_{v}\right) \exp \left(S / c_{v}\right)=0, \\
& \eta^{1} \partial S / \partial x+\eta^{2} \partial S / \partial y+\eta^{3} \partial S / \partial z+\xi_{1}^{10} V_{1}+\xi_{2}^{10} V_{2}+\xi_{3}^{10} V_{3}=0 .
\end{aligned}
$$

This system becomes the system of determining equations after using the fact that the original equations (51)-(54) are satisfied, and upon the explicit substitution of prolonged vector field coordinates (16).

The unknown quantities to be found are the tangent vector field coordinates

$$
\eta^{k}(\mathbf{u})=\left.\frac{\partial g^{k}(\mathbf{u}, a)}{\partial a}\right|_{a=0}, \quad k=1, \ldots, 10
$$

To ensure that the original equations (51)-(54) are satisfied, we use them to express the quantities

$$
\frac{\partial \Psi}{\partial x}, \frac{\partial \Psi}{\partial y}, \frac{\partial B_{3}}{\partial x}, \frac{\partial B_{3}}{\partial z}, \frac{\partial V_{1}}{\partial x}, \frac{\partial V_{2}}{\partial x}, \frac{\partial V_{3}}{\partial x}, \frac{\partial V_{3}}{\partial z}, \frac{\partial S}{\partial x}, \frac{\partial S}{\partial y}, \frac{\partial S}{\partial z}, P,
$$


and substitute them into (56)-(67).

Maple software shows that after setting the coefficients at different derivatives to zero in all twelve determining equations, one gets 187 dependent partial differential equations.

To be able to perform computations in reasonable time, we make a simplifying assumption, supposing that the tangent vector field coordinates (68) depend not on all variables $\mathbf{u}$, but only on some of them, as follows:

$$
\begin{aligned}
& \eta^{1}=\eta^{1}\left(V_{1}, \Psi\right), \eta^{2}=\eta^{2}\left(V_{2}, \Psi\right), \eta^{3}=\eta^{3}\left(V_{3}, \Psi\right) \\
& \eta^{4}=\eta^{4}\left(B_{1}\right), \eta^{5}=\eta^{5}\left(B_{2}\right), \eta^{6}=\eta^{6}\left(B_{3}\right) \\
& \eta^{7}=0, \eta^{8}=\eta^{8}(P), \eta^{9}=\eta^{9}(\rho, \Psi), \eta^{10}=\eta^{10}(\Psi)
\end{aligned}
$$

We remark that other choices of simplifying assumptions, when the functions $\eta^{k}$ depend no more than two variables, did not give more general results than the choice above.

Under the assumptions (69), the 187 equations mentioned above can be reduced (using Waterloo Maple with Rif package) to only ten independent equations, from which only two are partial differential equations, and the other eight are algebraic:

$$
\begin{aligned}
& \eta^{1}=\frac{V_{1}\left(\eta^{10} \rho+(\gamma-1) c_{v} \eta^{9}\right)}{2 c_{v} \rho}, \quad \eta^{2}=\frac{V_{2}\left(\eta^{10} \rho+(\gamma-1) c_{v} \eta^{9}\right)}{2 c_{v} \rho}, \\
& \eta^{3}=\frac{V_{3}\left(\eta^{10} \rho+(\gamma-1) c_{v} \eta^{9}\right)}{2 c_{v} \rho}, \quad \eta^{4}=\frac{B_{1}\left(\eta^{10} \rho+\gamma c_{v} \eta^{9}\right)}{2 c_{v} \rho}, \\
& \eta^{5}=\frac{B_{2}\left(\eta^{10} \rho+\gamma c_{v} \eta^{9}\right)}{2 c_{v} \rho}, \quad \eta^{6}=\frac{B_{3}\left(\eta^{10} \rho+\gamma c_{v} \eta^{9}\right)}{2 c_{v} \rho}, \quad \eta^{7}=0, \\
& \eta^{8}=\frac{P\left(\eta^{10} \rho+\gamma c_{v} \eta^{9}\right)}{c_{v} \rho}, \quad \frac{\partial \eta^{9}}{\partial \Psi}=-\frac{\partial \eta^{10}}{\partial \Psi} \frac{\rho}{\gamma c_{v}}, \quad \frac{\partial \eta^{9}}{\partial \rho}=\frac{\eta^{9}}{\rho} .
\end{aligned}
$$

The solution of the system (70) directly yields the infinitesimal operators (24), (25). This proves the part (ii) and so completes the proof of the theorem.

\section{B Alternative proof of Theorem 1}

(i) First, we prove that the operator $X^{(1)}$ is admissible for the system (1)-(2) in the case of incompressible plasma.

For the case $C=1$, we can write $b(\mathbf{r}), c(\mathbf{r})$ in the Bogoyavlenskij symmetries (5) as

$$
b(\mathbf{r})=\eta \cosh (\beta(\mathbf{r})), c(\mathbf{r})=\eta \sinh (\beta(\mathbf{r})) .
$$

Then for $\eta=1, a(\mathbf{r})=1$ the symmetries (5) become

$$
\begin{aligned}
& \mathbf{B}_{1}=\cosh (\beta(\mathbf{r})) \mathbf{B}+\sinh (\beta(\mathbf{r})) \sqrt{\mu \rho} \mathbf{V} \\
& \mathbf{V}_{1}=\frac{\sinh (\beta(\mathbf{r}))}{\sqrt{\mu \rho}} \mathbf{B}+\cosh (\beta(\mathbf{r})) \mathbf{V}
\end{aligned}
$$




$$
\rho_{1}=\rho, P_{1}=P+\left(\mathbf{B}^{2}-\mathbf{B}_{1}^{2}\right) /(2 \mu)
$$

these transformations have additive Lie group structure [2]. Writing $\beta(\mathbf{r})=\tau M(\mathbf{r}) / \sqrt{\mu \rho}$, and treating $\tau$ as a group parameter, we find according to (15):

$$
\begin{aligned}
& \xi^{i}(\mathbf{V}, \mathbf{B}, p, \rho)=0, \quad i=1,2,3 \\
& \left(\eta^{1}, \eta^{2}, \eta^{3}\right)=\left.\frac{\partial \mathbf{V}_{1}}{\partial \tau}\right|_{\tau=0}=\mathbf{B} \frac{M(\mathbf{r})}{\mu \rho} \\
& \left(\eta^{4}, \eta^{5}, \eta^{6}\right)=\left.\frac{\partial \mathbf{B}_{1}}{\partial \tau}\right|_{\tau=0}=\mathbf{V} M(\mathbf{r}) \\
& \eta^{7}=0, \quad \eta^{8}=\left.\frac{\partial P_{1}}{\partial \tau}\right|_{\tau=0}=-\frac{M(\mathbf{r})}{\mu}(\mathbf{V} \cdot \mathbf{B}) .
\end{aligned}
$$

This set of tangent vector field coordinates corresponds exactly to the infinitesimal operator $(20)$.

To get the infinitesimal operators (21) and (22) from Bogoyavlenskij symmetries (5), we take $b(\mathbf{r})=$ const $=\exp (\tau \delta), c(\mathbf{r})=0, a(\mathbf{r})=\exp (N(\mathbf{r}) \tau)$, where $N(\mathbf{r})$ is constant on magnetic field lines and streamlines and $\delta=$ const. Then $C=\exp (2 \tau \delta)$, and the symmetries become

$$
\begin{aligned}
& \mathbf{B}_{1}=\exp (\tau \delta) \mathbf{B} \\
& \mathbf{V}_{1}=\exp (\tau(\delta-N(\mathbf{r})) \mathbf{V}, \\
& \rho_{1}=\exp (2 \tau N(\mathbf{r})) \rho, \quad P_{1}=\exp (2 \tau \delta) P
\end{aligned}
$$

Treating $\tau$ as a group parameter, we find the corresponding infinitesimal operator

$$
X=(\delta-N(\mathbf{r})) \sum_{k=1}^{3} V_{k} \frac{\partial}{\partial V_{k}}+\delta \sum_{k=1}^{3} B_{k} \frac{\partial}{\partial B_{k}}+2 \delta P \frac{\partial}{\partial P}+2 N(\mathbf{r}) \rho \frac{\partial}{\partial \rho},
$$

which is a superposition of $X^{(2)}(21)$ and $X^{(3)}(22)$.

Finally, the operator (23) represents the shifts $P_{1}=P+C_{0}, C_{0}=$ const, and thus is evidently admissible by the MHD equilibrium equations, which depend only on the derivatives of pressure.

The commutator relations are given in Appendix A.

This proves part (i).

(ii) To prove that the operators (24), (25) are admitted by the compressible MHD equilibrium equations (1),(2),(4) for any density function, we take the transformations (7)-(8) with the following choice of parameters:

$$
a(\mathbf{r})=\exp (N(\mathbf{r}) \tau), b=\exp (\delta \tau)
$$

where $N(\mathbf{r})$ is a function constant on both magnetic field lines and streamlines. Then the formulas (7)-(8) become a Lie group with respect to addition in parameter $\tau$ :

$$
\rho_{1}=\exp (2 N(\mathbf{r}) \tau) \rho, \quad \mathbf{B}_{1}=\exp (\delta \tau) \mathbf{B}, \quad \mathbf{V}_{1}=\exp ((\delta-N(\mathbf{r})) \tau) \mathbf{V}
$$




$$
P_{1}=\exp (2 \delta \tau) P, \quad S_{1}=S+2 c_{v}(\delta-\gamma N(\mathbf{r})) \tau .
$$

The tangent vector field coordinates corresponding to this Lie group of transformations are found according to (15):

$$
\begin{aligned}
& \xi^{1}=\xi^{2}=\xi^{3}=0, \\
& \eta^{i}=V_{i}(\delta-N(\mathbf{r})), \quad \eta^{i+3}=B_{i} \delta, \quad i=1,2,3 \\
& \eta^{7}=2 N(\mathbf{r}) \rho, \quad \eta^{8}=2 \delta P, \quad \eta^{9}=2 c_{v}(\delta-\gamma N(\mathbf{r})) .
\end{aligned}
$$

These tangent vector field coordinates give rise to the infinitesimal operators (24) (set $N(\mathbf{r})=0$ ) and (25) (set $\delta=0$ ). This completes the proof of part (ii) of the theorem.

\section{Proof of Theorem 2}

The following lemma is necessary in the proof of Theorem 2.

Lemma 1 The incompressible MHD equilibrium system of equations (1)-(3) admits the discrete symmetries

$$
\mathbf{B}_{1}= \pm \mathbf{B}, \quad \mathbf{V}_{1}= \pm \mathbf{V}, \quad P_{1}=P, \quad \rho_{1}=\rho
$$

and

$$
\mathbf{B}_{1}=\mathbf{V} \sqrt{\mu \rho}, \quad \mathbf{V}_{1}=\mathbf{B} / \sqrt{\mu \rho}, \quad P_{1}=-P-\left(\mathbf{B}^{2}+\mathbf{V}^{2} \mu \rho\right) /(2 \mu), \quad \rho_{1}=\rho .
$$

Compressible ideal MHD equilibrium equations (1)-(2) with ideal gas state equation (4), for arbitrary density, admit the discrete symmetries (74).

\section{Proof of the Lemma.}

The proof is based on the complexification of parameters of known continuous point symmetries of the systems under consideration.

First we consider the incompressible MHD equilibrium system (1)-(3). By Theorem 1, it admits the infinitesimal operators $X^{(1)}, X^{(2)}, X^{(3)}(20)-(22)$ and therefore the continuous Lie point transformations (27)-(29).

If we take an equilibrium configuration $\{\mathbf{V}, \mathbf{B}, P, \rho\}$ and apply the transformation (28) with $\tau=\tau_{1}$ and then (29) with $\tau=\tau_{2}$, we get a new solution

$$
\rho_{1}=\exp \left(2 N(\mathbf{r}) \tau_{2}\right) \rho, \quad \mathbf{B}_{1}=\exp \left(\tau_{1}\right) \mathbf{B}, \quad \mathbf{V}_{1}=\exp \left(\tau_{1}-N(\mathbf{r}) \tau_{2}\right) \mathbf{V}, \quad P_{1}=P .
$$

Now, using the combinations $\left\{\tau_{1}=\pi i, N(\mathbf{r}) \tau_{2}=0\right\},\left\{\tau_{1}=\pi i, N(\mathbf{r}) \tau_{2}=\pi i\right\},\left\{\tau_{1}=\right.$ $\left.0, N(\mathbf{r}) \tau_{2}=-\pi i\right\}$, we get all transformations (74), with $\rho_{1}=\rho$.

To prove that the discrete symmetry $(75)$ is admissible, we take an equilibrium configuration $\{\mathbf{V}, \mathbf{B}, P, \rho\}$ and apply first the transformation (27) with $M(\mathbf{r}) \tau=\sqrt{\mu \rho} \pi i / 2$, and then the 
transformation (28) with $\tau=-\pi i / 2$. The final result is real and coincides with the required formula (75). The density $\rho$ is not transformed.

The existence of the refection symmetry (74) for the compressible case is proven the same way as for incompressible, using the operators (24)-(25) and the corresponding transformations (31)-(32). Lemma is proven.

\section{Proof of Theorem 2.}

(i). In the case $C>0$, we denote $C=q^{2}, \eta= \pm 1, \sigma= \pm 1, \lambda= \pm 1$, and write $a(\mathbf{r}), b(\mathbf{r}), c(\mathbf{r})$ in Bogoyavlenskij symmetries (5) as

$$
a(\mathbf{r})=\eta \exp (N(\mathbf{r})), b(\mathbf{r})=\sigma q \cosh (\beta(\mathbf{r})), c(\mathbf{r})=\lambda q \sinh (\beta(\mathbf{r})) .
$$

Therefore the transformations (5) become

$$
\begin{aligned}
& \mathbf{B}_{1}=q \sigma \cosh (\beta(\mathbf{r})) \mathbf{B}+q \lambda \sinh (\beta(\mathbf{r})) \sqrt{\mu \rho} \mathbf{V}, \\
& \mathbf{V}_{1}=q \lambda \frac{\sinh (\beta(\mathbf{r}))}{\eta \exp (N(\mathbf{r})) \sqrt{\mu \rho}} \mathbf{B}+q \sigma \frac{\cosh (\beta(\mathbf{r}))}{\eta \exp (N(\mathbf{r}))} \mathbf{V}, \\
& \rho_{1}=\exp (2 N(\mathbf{r})) \rho, P_{1}=q^{2} P+\left(q^{2} \mathbf{B}^{2}-\mathbf{B}_{1}^{2}\right) /(2 \mu),
\end{aligned}
$$

From the original solution $\{\mathbf{V}, \mathbf{B}, P, \rho\}$, using appropriately the reflections (74) and the mixing transformations (27), we can obtain a solution

$$
\begin{aligned}
& \tilde{\mathbf{B}}_{1}=\sigma \cosh (\beta(\mathbf{r})) \mathbf{B}+\lambda \sinh (\beta(\mathbf{r})) \sqrt{\mu \rho} \mathbf{V}, \\
& \tilde{\mathbf{V}}_{1}=\lambda \frac{\sinh (\beta(\mathbf{r}))}{\eta \sqrt{\mu \rho}} \mathbf{B}+\sigma \frac{\cosh (\beta(\mathbf{r}))}{\eta} \mathbf{V}, \\
& \tilde{\rho}_{1}=\rho, \tilde{P}_{1}=P+\left(\mathbf{B}^{2}-\tilde{\mathbf{B}}_{1}^{2}\right) /(2 \mu) .
\end{aligned}
$$

This intermediate solution can be scaled by applying (28) with $\tau=\ln q$ and (29) with $\tau=1$ to obtain (76).

In the case $C<0$, we denote $C=-q^{2}, \eta= \pm 1, \sigma= \pm 1, \lambda= \pm 1$. Then

$$
a(\mathbf{r})=\eta \exp (N(\mathbf{r})), b(\mathbf{r})=\sigma q \sinh (\beta(\mathbf{r})), c(\mathbf{r})=\lambda q \cosh (\beta(\mathbf{r})) .
$$

Therefore the transformations (5) can be written as

$$
\begin{aligned}
& \mathbf{B}_{1}=q \sigma \sinh (\beta(\mathbf{r})) \mathbf{B}+q \lambda \cosh (\beta(\mathbf{r})) \sqrt{\mu \rho} \mathbf{V}, \\
& \mathbf{V}_{1}=q \lambda \frac{\cosh (\beta(\mathbf{r}))}{\eta \exp (N(\mathbf{r})) \sqrt{\mu \rho}} \mathbf{B}+q \sigma \frac{\sinh (\beta(\mathbf{r}))}{\eta \exp (N(\mathbf{r}))} \mathbf{V}, \\
& \rho_{1}=\exp (2 N(\mathbf{r})) \rho, P_{1}=-q^{2} P-\left(q^{2} \mathbf{B}^{2}+\mathbf{B}_{1}^{2}\right) /(2 \mu)
\end{aligned}
$$

We will show that this transform can be found from an original solution by combining (27)-(29), (74), (75). 
First, to the original solution $\{\mathbf{V}, \mathbf{B}, P, \rho\}$ we apply (75) to obtain

$$
\mathbf{B}_{2}=\mathbf{V} \sqrt{\mu \rho}, \quad \mathbf{V}_{2}=\mathbf{B} / \sqrt{\mu \rho}, \quad P_{2}=-P-\left(\mathbf{B}^{2}+\mathbf{V}^{2} \mu \rho\right) /(2 \mu), \quad \rho_{2}=\rho .
$$

In the way described above in this proof and using (27)-(29) and (74), the solution $\left\{\mathbf{V}_{2}, \mathbf{B}_{2}, P_{2}, \rho_{2}\right\}$ can be transformed to another solution

$$
\begin{aligned}
& \mathbf{B}_{3}=q \lambda \cosh (\beta(\mathbf{r})) \mathbf{B}_{2}+q \sigma \sinh (\beta(\mathbf{r})) \sqrt{\mu \rho_{2}} \mathbf{V}_{2}, \\
& \mathbf{V}_{3}=q \sigma \frac{\sinh (\beta(\mathbf{r}))}{\eta \exp (N(\mathbf{r})) \sqrt{\mu \rho_{2}}} \mathbf{B}_{2}+q \lambda \frac{\cosh (\beta(\mathbf{r}))}{\eta \exp (N(\mathbf{r}))} \mathbf{V}_{2}, \\
& \rho_{3}=\exp (2 N(\mathbf{r})) \rho_{2}, P_{3}=q^{2} P_{2}+\left(q^{2} \mathbf{B}_{2}^{2}-\mathbf{B}_{3}^{2}\right) /(2 \mu),
\end{aligned}
$$

After the substitution of $\left\{\mathbf{V}_{2}, \mathbf{B}_{2}, P_{2}, \rho_{2}\right\}$, the new solution (80) coincides with the desired form (78).

The fact that the operators (20)-(22), and thus the corresponding transformations (27)(29), can be obtained from Bogoyavlenskij symmetries (5) is proven in Appendix B. This proves part (i).

(ii). Consider the Bogoyavlenskij symmetries (7)-(8) for compressible MHD equilibria with $b \geq 0, a(\mathbf{r})>0$. To an equilibrium $\{\mathbf{V}, \mathbf{B}, P, \rho, S\}$ we apply the Lie point transformations (31) with $\tau=\ln b$ and (32) with $\{\tau=1, N(\mathbf{r})=\ln a(\mathbf{r})\}$. This converts the original solution exactly into the form (7)-(8).

Suppose now that $b \leq 0, a(\mathbf{r})>0$. Let $\{\mathbf{V}, \mathbf{B}, P, \rho, S\}$ be an MHD equilibrium. Then by applying to it first (74) in the form $\mathbf{B} \rightarrow-\mathbf{B}, \quad \mathbf{V} \rightarrow-\mathbf{V}$, and then the Lie symmetries (31) with $\tau=\ln |b|$ and (32) with $\{\tau=1, N(\mathbf{r})=\ln a(\mathbf{r})\}$ we obtain

$$
\begin{aligned}
& \rho_{1}=a^{2}(\mathbf{r}) \rho, \quad \mathbf{B}_{1}=b \mathbf{B}, \quad \mathbf{V}_{1}=\frac{b}{a(\mathbf{r})} \mathbf{V}, \quad P_{1}=b^{2} P, \\
& S_{1}=S+2 c_{v}(\ln |b|-\gamma \ln |a(\mathbf{r})|) .
\end{aligned}
$$

This form coincides with the transform (7)-(8).

The cases when $a(\mathbf{r})$ can be negative are treated in the same way. In the points where $a(\mathbf{r})<0$, an additional reflection transformation (74) in the form $\mathbf{B} \rightarrow \mathbf{B}, \quad \mathbf{V} \rightarrow-\mathbf{V}$ needs to be applied to the original solution.

Thus the composition of Lie symmetries (31) and (32) yields the transformation (7)-(8) of compressible MHD equilibria.

Conversely, the operators (24)-(25), and so the transformations (31) and (32), the are implied by Bogoyavlenskij symmetries (7)-(8), as shown in Appendix B.

This proves part (ii) and completes the proof of Theorem 2. 


\section{References}

[1] Bogoyavlenskij O. I. Phys. Lett. A. 291 (4-5), 256-264 (2001).

[2] Bogoyavlenskij O. I. Phys. Rev. E. 66 (5), art. no. 056410 (2002).

[3] Olver P. J. Applications of Lie groups to differential equations. New York: SpringerVerlag (1993).

[4] CRC handbook of Lie group analysis of differential equations. (Editor: N.H. Ibragimov). Boca Raton, Fl.: CRC Press (1993).

[5] Hydon P.E. Proc. Roy. Soc. Lond. A 454, 1961-197270 (1998).

[6] Hydon P.E. Eur. J. Appl. Math. 11, 515-527 (2000).

[7] Hydon P.E. Contemp. Math. 285, 61-70 (2001).

[8] Mansfield E.L. Differential Groebner Bases. Ph.D. diss., University of Sydney (1991)

[9] Ollivier F. Standard Bases of Differential Ideals. Lecture Notes in Comp. Sci. 508, 304-321 (1991).

[10] Reid G.J., Wittkopf A.D., and Boulton A. Eur. J. Appl. Math. 7, 604-635 (1996).

[11] Reid G.J., Wittkopf A.D. Proc. ISSAC 2000. 272-280, ACM Press (2000).

[12] Boulier F., Lazard D., Ollivier F. and Petitot M. Proc. ISAAC'95. ACM Press (1995).

[13] Hubert E. J. Symb. Comput. 29, 641-662 (2000).

[14] Hereman W. Euromath Bull. 1, 45-79 (1994).

[15] Reid G.J., Wittkopf A.D. The Long Guide to the Standard Form Package. 1993. Programs and documentation available on the web at http://www.cecm.sfu.ca/ ${ }^{\sim}$ wittkopf.

[16] Mansfield E.L. diffgrob2: A symbolic algebra package for analysing systems of PDE usig Maple. ftp://ftp.ukc.ac.uk, directory: pub/maths/liz .

[17] Tahenbaum B.S. Plasma Physics. McGraw-Hill (1967).

[18] Kruskal M.D., Kulsrud R.M. Phys. Fluids. 1, 265 (1958). 